\title{
Stability for the Mixed Type of Quartic and Quadratic Functional Equations
}

\author{
Young-Su Lee, Soomin Kim, and Chaewon Kim \\ Hana Academy Seoul, Seoul 121-741, Republic of Korea \\ Correspondence should be addressed to Young-Su Lee; masuri@kaist.ac.kr
}

Received 18 April 2014; Revised 21 July 2014; Accepted 4 August 2014; Published 17 August 2014

Academic Editor: Liviu Cădariu

Copyright (C) 2014 Young-Su Lee et al. This is an open access article distributed under the Creative Commons Attribution License, which permits unrestricted use, distribution, and reproduction in any medium, provided the original work is properly cited.

\begin{abstract}
We establish the general solutions of the following mixed type of quartic and quadratic functional equation: $f(2 x+y)+f(2 x-y)=$ $4 f(x+y)+4 f(x-y)+2 f(2 x)-8 f(x)-6 f(y)$. Moreover we prove the Hyers-Ulam-Rassias stability of this equation under the approximately quartic and the approximately quadratic conditions.
\end{abstract}

\section{Introduction}

The stability problems of functional equations go back to 1940, when Ulam [1] proposed the following problem concerning group homomorphisms.

Let $G_{1}$ be a group and let $G_{2}$ be a metric group with metric $d(\cdot, \cdot)$ and $\epsilon$ a positive number. Does there exist a positive $\delta$ such that for every $f$ : $G_{1} \rightarrow G_{2}$ with $d(f(x y), f(x) f(y)) \leq \epsilon$ there exists a group homomorphism $L: G_{1} \rightarrow G_{2}$ such that $d(f(x), L(x))<\delta$ for all $x$ in $G_{1}$ ?

In 1941, Hyers [2] had affirmatively answered the question of Ulam for Banach spaces. He proved that if $f$ is a mapping between Banach spaces satisfying $\|f(x+y)-f(x)-f(y)\| \leq \epsilon$ for some fixed $\epsilon \geq 0$, then there exists the unique additive mapping $A$ such that $\|f(x)-A(x)\| \leq \epsilon$. Actually, the additive mapping $A$ is explicitly constructed from the given function $f$ by the formular

$$
A(x)=\lim _{n \rightarrow \infty} 2^{-n} f\left(2^{n} x\right) \quad \text { or } \quad A(x)=\lim _{n \rightarrow \infty} 2^{n} f\left(2^{-n} x\right) .
$$

This method is called a direct method. The theorem of Hyers was generalized by Aoki [3] for additive mappings and by Rassias [4] for linear mappings by considering an unbounded Cauchy difference to be controlled by $\epsilon\left(\|x\|^{p}+\|y\|^{p}\right)$. In addition, Rassias generalized the Hyers' stability result by introducing two weaker conditions controlled by the product of different powers of norms and mixed product-sum of powers of norms, respectively (see [5-9]). In 1994, Găvruța [10] gave a generalization of Rassias theorem by replacing $\epsilon\left(\|x\|^{p}+\|y\|^{p}\right)$ by a general control function $\phi(x, y)$. Instead of the direct method, Cădariu and Radu [11] introduced another approach for proving the stability of functional equations (see also [12]) via the fixed point theory. They observed that the existence of a solution $A$ of the functional equation and the estimation of the difference with the given mapping $f$ can be obtained from the fixed point alternative. This method is called a fixed point method.

As of now, both the direct method and the fixed point method have been intensively used in the study of stability problems of various types of functional equations (see [1319]). In particular, one of the important functional equations studied is the quadratic functional equation:

$$
f(x+y)+f(x-y)=2 f(x)+2 f(y) .
$$

We note that the quadratic function $f(x)=x^{2}$ is a solution of (2). So one usually calls the above functional equation quadratic and every solution of (2) is said to be a quadratic mapping. Stability results of quadratic functional equations can be found in [20-22]. On the other hand, Rassias [23] investigated stability problems of the following functional equation:

$$
\begin{aligned}
& f(x+2 y)+f(x-2 y)+6 f(x) \\
& \quad=4 f(x+y)+4 f(x-y)+24 f(y) .
\end{aligned}
$$


It is easy to see that $f(x)=x^{4}$ is a solution of (3) by virtue of the algebraic identity

$$
\begin{aligned}
(x & +2 y)^{4}+(x-2 y)^{4}+6 x^{4} \\
& =4(x+y)^{4}+4(x-y)^{4}+24 y^{4} .
\end{aligned}
$$

For this reason, (3) is called a quartic functional equation and every solution of (3) is said to be a quartic mapping. Chung and Sahoo [24] determined the general solutions of (3) without assuming any regularity conditions on the unknown function. In fact, they proved that the function $f$ : $\mathbb{R} \rightarrow \mathbb{R}$ is a solution of (3) if and only if $f(x)=A(x, x, x, x)$, where the function $A: \mathbb{R}^{4} \rightarrow \mathbb{R}$ is symmetric and additive in each variable. Since the solution of (3) is even, we can rewrite (3) as

$$
\begin{aligned}
& f(2 x+y)+f(2 x-y) \\
& \quad=4 f(x+y)+4 f(x-y)+24 f(x)-6 f(y) .
\end{aligned}
$$

Lee et al. [25] obtained the general solutions of (5) and proved the Hyers-Ulam-Rassias stability of this equation (see also [26]). Lee and Chung [27] proved the stability of the following quartic functional equation, which is a generalization of (5),

$$
\begin{aligned}
f(n x+y)+f(n x-y) \\
=n^{2} f(x+y)+n^{2} f(x-y)+2 n^{2}\left(n^{2}-1\right) f(x) \\
\quad-2\left(n^{2}-1\right) f(y)
\end{aligned}
$$

for fixed integer $n$ with $n \neq 0, \pm 1$. Also Kim [28] solved the general solutions and proved the Hyers-Ulam-Rassias stability for the mixed type of quartic and quadratic functional equation:

$$
\begin{aligned}
f\left(x_{1}\right. & \left.+x_{2}+x_{3}\right)+f\left(x_{1}+x_{2}-x_{3}\right)+f\left(x_{1}-x_{2}+x_{3}\right) \\
& +f\left(x_{1}-x_{2}-x_{3}\right)+4 f\left(x_{1}\right)+4 f\left(x_{2}\right)+4 f\left(x_{3}\right) \\
= & 2 f\left(x_{1}+x_{2}\right)+2 f\left(x_{1}-x_{2}\right)+2 f\left(x_{1}+x_{3}\right) \\
& +2 f\left(x_{1}-x_{3}\right)+2 f\left(x_{2}+x_{3}\right)+2 f\left(x_{2}-x_{3}\right) .
\end{aligned}
$$

Gordji et al. [29] introduced another mixed type of quartic and quadratic functional equation:

$$
\begin{aligned}
f(n x+y)+f(n x-y) \\
=n^{2} f(x+y)+n^{2} f(x-y)+2\left(f(n x)-n^{2} f(x)\right) \\
\quad-2\left(n^{2}-1\right) f(y)
\end{aligned}
$$

for fixed integers $n$ with $n \neq 0, \pm 1$. They established the general solutions and proved the Hyers-Ulam-Rassias stability of this equation in quasi-Banach spaces.

In this paper, we deal with the following mixed type of quartic and quadratic functional equations, for the case $n=2$ in (8),

$$
\begin{aligned}
& f(2 x+y)+f(2 x-y) \\
& \quad=4 f(x+y)+4 f(x-y)+2 f(2 x)-8 f(x)-6 f(y) .
\end{aligned}
$$

In Section 2, we solve the general solutions of (9) using another way as in [29]. As a matter of fact, $f$ satisfies (9) if and only if there exists a quartic mapping $T$ and a quadratic mapping $Q$ which satisfy (5) and (2), respectively; the mapping $f$ can be written as $f(x)=T(x)+Q(x)$. Using the idea of Gãvruta [10] we prove the Hyers-UlamRassias stability of (9) in Section 3. Applying the different approaches as in [29] we prove the Hyers-Ulam-Rassias stability of (9) under the approximately quartic condition and the approximately quadratic condition in Sections 4 and 5, respectively.

\section{General Solutions of (9)}

Throughout this section, we denote both $X$ and $Y$ by real vector spaces.

It is well-known [30] that a mapping $f: X \rightarrow Y$ satisfies the quadratic functional equation (2) if and only if there exists a unique symmetric biadditive mapping $B$ such that $f(x)=$ $B(x, x)$ for all $x \in X$. The biadditive mapping $B$ is given by

$$
B(x, y)=\frac{1}{4}[f(x+y)-f(x-y)]
$$

Similarly, a mapping $f: X \rightarrow Y$ satisfies the quartic functional equation (5) if and only if there exists a symmetric biquadratic mapping $F: X \times X \rightarrow Y$ such that $f(x)=F(x, x)$ for all $x \in X$ (see [25]). The biquadratic mapping $F$ is given by

$$
F(x, y)=\frac{1}{12}[f(x+y)+f(x-y)-2 f(x)-2 f(y)]
$$

Now we are going to establish the general solutions of (9).

Lemma 1. If a mapping $f: X \rightarrow Y$ satisfies (9), then the mapping $g: X \rightarrow Y$ defined by $g(x)=f(2 x)-4 f(x)$ is a quartic mapping satisfying (5).

Proof. Putting $x=y=0$ in (9) gives $f(0)=0$. Letting $x=0$ in (9) we have $f(-y)=f(y)$ for all $y \in X$. Substituting $y$ by $x$ in (9) yields

$$
f(3 x)=6 f(2 x)-15 f(x)
$$

for all $x \in X$. Replacing $y$ by $2 x$ in (9) and using the evenness of $f$ we obtain

$$
f(4 x)=4 f(3 x)-4 f(2 x)-4 f(x)
$$

for all $x \in X$. Combining (12) and (13) to eliminate the term $f(3 x)$ gives

$$
f(4 x)=20 f(2 x)-64 f(x)
$$


for all $x \in X$. By the definition of $g$ and using (9), (14) we have

$$
\begin{aligned}
& g(2 x+y)+g(2 x-y) \\
&= {[f(4 x+2 y)+f(4 x-2 y)] } \\
&-4[f(2 x+y)+f(2 x-y)] \\
&= {[4 f(2 x+2 y)+4 f(2 x-2 y)} \\
&+2 f(4 x)-8 f(2 x)-6 f(2 y)] \\
&- 4[4 f(x+y)+4 f(x-y) \\
&\quad+2 f(2 x)-8 f(x)-6 f(y)] \\
&= 4[f(2 x+2 y)-4 f(x+y)] \\
&+ 4[f(2 x-2 y)-4 f(x-y)] \\
&+ 24[f(2 x)-4 f(x)]-6[f(2 y)-4 f(y)] \\
&= 4 g(x+y)+4 g(x-y)+24 g(x)-6 g(y)
\end{aligned}
$$

for all $x, y \in X$. This shows that $g$ satisfies (5).

Lemma 2. If a mapping $f: X \rightarrow Y$ satisfies (9), then the mapping $h: X \rightarrow Y$ defined by $h(x)=f(2 x)-16 f(x)$ is a quadratic mapping satisfying (2).

Proof. Interchanging the role of $x$ and $y$ in (9) and using the evenness of $f$ we have

$$
\begin{aligned}
& f(x+2 y)+f(x-2 y) \\
& \quad=4 f(x+y)+4 f(x-y)+2 f(2 y)-8 f(y)-6 f(x)
\end{aligned}
$$

for all $x, y \in X$. Putting $y=2 y$ in (9) and using (16) we figure out

$$
\begin{aligned}
& f(2 x+2 y)+f(2 x-2 y) \\
&=4 f(x+2 y)+4 f(x-2 y) \\
&+ 2 f(2 x)-8 f(x)-6 f(2 y) \\
&= 4[4 f(x+y)+4 f(x-y) \\
&+2 f(2 y)-8 f(y)-6 f(x)] \\
&+ 2 f(2 x)-8 f(x)-6 f(2 y) \\
&= 16[f(x+y)+f(x-y)]+2[f(2 x)-16 f(x)] \\
&+2[f(2 y)-16 f(y)]
\end{aligned}
$$

for all $x, y \in X$. This shows that $h$ satisfies (2).

From the preceding Lemmas we establish the general solutions of (9) as follows.

Theorem 3. A mapping $f: X \rightarrow Y$ satisfies (9) for all $x, y \in$ $X$ if and only if there exists a symmetric biquartic mapping $F$ : $X \times X \rightarrow Y$ and a symmetric biadditive mapping $B: X \times X \rightarrow$ $Y$ such that $f(x)=F(x, x)+B(x, x)$ for all $x \in X$.
Proof. We assume that the mapping $f: X \rightarrow Y$ satisfies (9). Define mappings $g, h: X \rightarrow Y$ by

$$
g(x)=f(2 x)-16 f(x), \quad h(x)=f(2 x)-4 f(x)
$$

for all $x \in X$. By Lemmas 1 and 2 we note that the mappings $g$ and $h$ satisfy (5) and (2), respectively, and

$$
f(x)=-\frac{1}{12} g(x)+\frac{1}{12} h(x)
$$

for all $x \in X$. According to the results as in $[25,30]$ there exists a symmetric biquadratic mapping $F: X \times X \rightarrow Y$ and a symmetric biadditive mapping $B: X \times X \rightarrow Y$ such that

$$
g(x)=-12 F(x, x), \quad h(x)=12 B(x, x)
$$

for all $x \in X$. Conversely, one can easily verify that the mappings $F$ and $B$ satisfy (9) by a simple computation.

\section{Stability of (9)}

Now we are going to prove the Hyers-Ulam-Rassias stability for the mixed type quartic and quadratic functional equation. In what follows, we denote $X$ by a real vector space and $Y$ by a Banach space. Let $\mathbb{R}^{+}$denote the set of all nonnegative real numbers and $\mathbb{N}$ the set of all positive integers. For convenience, we define the difference operator for a given mapping $f: X \rightarrow Y$ by

$$
\begin{aligned}
D f(x, y)= & f(2 x+y)+f(2 x-y)-4 f(x+y) \\
& -4 f(x-y)-2 f(2 x)+8 f(x)+6 f(y)
\end{aligned}
$$

for all $x, y \in X$.

Theorem 4. Let $\phi: X \times X \rightarrow \mathbb{R}^{+}$be a mapping satisfying

$$
\sum_{i=0}^{\infty} \frac{\phi\left(2^{i} x, 2^{i} y\right)}{4^{i}}<\infty
$$

for all $x, y \in X$. If a mapping $f: X \rightarrow Y$ with $f(0)=0$ satisfies

$$
\|D f(x, y)\| \leq \phi(x, y)
$$

for all $x, y \in X$, then there exists a quartic and quadratic mapping $H: X \rightarrow Y$ such that

$$
\|f(x)-H(x)\| \leq \frac{4}{3} \sum_{i=0}^{\infty}\left(\frac{1}{4^{i}}-\frac{1}{4^{2 i+1}}\right) \Phi\left(2^{i} x\right)
$$

for all $x \in X$, where the mapping $\Phi$ is given by

$$
\Phi(x)=\frac{1}{64}\left[\phi(x, 2 x)+4 \phi(x, x)+\frac{4}{3} \phi(0, x)\right]
$$

for all $x \in X$. The mapping $H$ is given by

$$
\begin{aligned}
H(x)=\lim _{n \rightarrow \infty} & {\left[\frac{4}{3}\left(\frac{1}{4^{n}}-\frac{1}{4^{2 n+1}}\right) f\left(2^{n} x\right)\right.} \\
& \left.-\frac{1}{12}\left(\frac{1}{4^{n}}-\frac{1}{4^{2 n}}\right) f\left(2^{n+1} x\right)\right]
\end{aligned}
$$

for all $x \in X$. 
Proof. Putting $x=0$ and then replacing $y$ by $x$ in (23), one has the approximately even condition of $f$ as follows:

$$
\|f(x)-f(-x)\| \leq \frac{1}{3} \phi(0, x)
$$

for all $x \in X$. Substituting $y$ by $x$ in (23) gives

$$
\|f(3 x)-6 f(2 x)+15 f(x)\| \leq \phi(x, x)
$$

for all $x \in X$. Replacing $y$ by $2 x$ in (23) yields

$$
\|f(4 x)-4 f(3 x)+4 f(2 x)+8 f(x)-4 f(-x)\| \leq \phi(x, 2 x)
$$

for all $x \in X$. Combining (27), (28), and (29) to eliminate the terms $f(-x)$ and $f(3 x)$ we have the following relation

$$
\left\|f(x)-\frac{5}{16} f(2 x)+\frac{1}{64} f(4 x)\right\| \leq \Phi(x)
$$

for all $x \in X$.

Making use of induction arguments in (30) we obtain

$$
\begin{aligned}
\left\|f(x)-\frac{4}{3}\left(\frac{1}{4^{n}}-\frac{1}{4^{2 n+1}}\right) f\left(2^{n} x\right)+\frac{1}{12}\left(\frac{1}{4^{n}}-\frac{1}{4^{2 n}}\right) f\left(2^{n+1} x\right)\right\| \\
\leq \frac{4}{3} \sum_{i=0}^{n-1}\left(\frac{1}{4^{i}}-\frac{1}{4^{2 i+1}}\right) \Phi\left(2^{i} x\right)
\end{aligned}
$$

for all $n \in \mathbb{N}$ and for all $x \in X$. Actually (30) proves the validity of the inequality (31) for the case $n=1$. Assume that inequality (31) holds for some $n \in \mathbb{N}$. Using (30) and (31) we have the following relation

$$
\begin{aligned}
\| f(x) & -\frac{4}{3}\left(\frac{1}{4^{n+1}}-\frac{1}{4^{2 n+3}}\right) f\left(2^{n+1} x\right) \\
+ & \frac{1}{12}\left(\frac{1}{4^{n+1}}-\frac{1}{4^{2 n+2}}\right) f\left(2^{n+2} x\right) \| \\
\leq \| & f(x)-\frac{4}{3}\left(\frac{1}{4^{n}}-\frac{1}{4^{2 n+1}}\right) f\left(2^{n} x\right) \\
& +\frac{1}{12}\left(\frac{1}{4^{n}}-\frac{1}{4^{2 n}}\right) f\left(2^{n+1} x\right) \| \\
& +\frac{4}{3}\left(\frac{1}{4^{n}}-\frac{1}{4^{2 n+1}}\right) \\
& \times\left\|f\left(2^{n} x\right)-\frac{5}{16} f\left(2^{n+1} x\right)+\frac{1}{64} f\left(2^{n+2} x\right)\right\| \\
\leq & \frac{4}{3} \sum_{i=0}^{n-1}\left(\frac{1}{4^{i}}-\frac{1}{4^{2 i+1}}\right) \Phi\left(2^{i} x\right)+\frac{4}{3}\left(\frac{1}{4^{n}}-\frac{1}{4^{2 n+1}}\right) \Phi\left(2^{n} x\right)
\end{aligned}
$$

for all $x \in X$ and $n \in \mathbb{N}$. This proves the validity of inequality (31) for the case $n+1$.

Now let us define a sequence $\left\{f_{n}(x)\right\}$ by

$$
\begin{aligned}
f_{n}(x)= & \frac{4}{3}\left(\frac{1}{4^{n}}-\frac{1}{4^{2 n+1}}\right) f\left(2^{n} x\right) \\
& -\frac{1}{12}\left(\frac{1}{4^{n}}-\frac{1}{4^{2 n}}\right) f\left(2^{n+1} x\right)
\end{aligned}
$$

and claim that it is a convergent sequence. For any integers $m, n$ with $m>n \geq 0$, we verify by (30) that

$$
\begin{aligned}
& \left\|f_{m}(x)-f_{n}(x)\right\| \\
& \leq \sum_{k=n}^{m-1}\left\|f_{k+1}(x)-f_{k}(x)\right\| \\
& =\sum_{k=n}^{m-1} \frac{4}{3}\left(\frac{1}{4^{k}}-\frac{1}{4^{2 k+1}}\right) \\
& \quad \times\left\|f\left(2^{k} x\right)-\frac{5}{16} f\left(2^{k+1} x\right)+\frac{1}{64} f\left(2^{k+2} x\right)\right\| \\
& \leq \sum_{k=n}^{m-1} \frac{4}{3}\left(\frac{1}{4^{k}}-\frac{1}{4^{2 k+1}}\right) \Phi\left(2^{k} x\right)
\end{aligned}
$$

for all $x \in X$. Since the right-hand side of the above inequality tends to 0 as $n \rightarrow \infty$ by assumption, the sequence $\left\{f_{n}(x)\right\}$ is a Cauchy sequence in $Y$. Thus, we may define a mapping $H: X \rightarrow Y$ by

$$
\begin{aligned}
H(x)=\lim _{n \rightarrow \infty}[ & \frac{4}{3}\left(\frac{1}{4^{n}}-\frac{1}{4^{2 n+1}}\right) f\left(2^{n} x\right) \\
& \left.-\frac{1}{12}\left(\frac{1}{4^{n}}-\frac{1}{4^{2 n}}\right) f\left(2^{n+1} x\right)\right]
\end{aligned}
$$

for all $x \in X$. By virtue of the inequality (23) we figure out

$$
\begin{aligned}
&\left\|D f_{n}(x, y)\right\| \\
& \leq \frac{4}{3}\left(\frac{1}{4^{n}}-\frac{1}{4^{2 n+1}}\right)\left\|D f\left(2^{n} x, 2^{n} y\right)\right\| \\
&+\frac{1}{12}\left(\frac{1}{4^{n}}-\frac{1}{4^{2 n}}\right)\left\|D f\left(2^{n+1} x, 2^{n+1} y\right)\right\| \\
& \leq \frac{4}{3}\left(\frac{1}{4^{n}}-\frac{1}{4^{2 n+1}}\right) \phi\left(2^{n} x, 2^{n} y\right) \\
&+\frac{1}{12}\left(\frac{1}{4^{n}}-\frac{1}{4^{2 n}}\right) \phi\left(2^{n+1} x, 2^{n+1} y\right)
\end{aligned}
$$

for all $n \in \mathbb{N}$ and for all $x, y \in X$. Letting $n \rightarrow \infty$ in the above inequality we see that

$$
D H(x, y)=0
$$

which shows that $H$ satisfies (9). Finally letting $n \rightarrow \infty$ in (31) we have the result (24). This completes the proof.

Theorem 5. Let $\phi: X \times X \rightarrow \mathbb{R}^{+}$be a mapping satisfying

$$
\sum_{i=0}^{\infty} 16^{i} \phi\left(\frac{x}{2^{i}}, \frac{y}{2^{i}}\right)<\infty
$$

for all $x, y \in X$. If a mapping $f: X \rightarrow Y$ satisfies

$$
\|D f(x, y)\| \leq \phi(x, y)
$$

for all $x, y \in X$, then there exists a quartic and quadratic mapping $H: X \rightarrow Y$ such that

$$
\|f(x)-H(x)\| \leq \frac{64}{3} \sum_{i=0}^{\infty}\left(4^{2 i+1}-4^{i}\right) \Phi\left(\frac{x}{2^{i+2}}\right)
$$


for all $x \in X$. The mapping $H$ is given by

$$
\begin{aligned}
H(x)=\lim _{n \rightarrow \infty}[ & \frac{1}{3}\left(4^{2 n+1}-4^{n}\right) f\left(\frac{x}{2^{n}}\right) \\
& \left.-\frac{16}{3}\left(4^{2 n}-4^{n}\right) f\left(\frac{x}{2^{n+1}}\right)\right]
\end{aligned}
$$

for all $x \in X$.

Proof. Replacing $x$ by $x / 4$ in (30) we have

$$
\left\|f(x)-20 f\left(\frac{x}{2}\right)+64 f\left(\frac{x}{4}\right)\right\| \leq 64 \Phi\left(\frac{x}{4}\right)
$$

for all $x \in X$. Using the induction argument in (42) we obtain

$$
\begin{aligned}
& \left\|f(x)-\frac{1}{3}\left(4^{2 n+1}-4^{n}\right) f\left(\frac{x}{2^{n}}\right)+\frac{16}{3}\left(4^{2 n}-4^{n}\right) f\left(\frac{x}{2^{n+1}}\right)\right\| \\
& \leq \frac{64}{3} \sum_{i=0}^{n-1}\left(4^{2 i+1}-4^{i}\right) \Phi\left(\frac{x}{2^{i+2}}\right)
\end{aligned}
$$

for all $n \in \mathbb{N}$ and for all $x \in X$. We define a sequence $\left\{f_{n}(x)\right\}$ by

$$
f_{n}(x)=\frac{1}{3}\left(4^{2 n+1}-4^{n}\right) f\left(\frac{x}{2^{n}}\right)-\frac{16}{3}\left(4^{2 n}-4^{n}\right) f\left(\frac{x}{2^{n+1}}\right)
$$

and show that it is a Cauchy sequence. For any integers $m, n$ with $m>n \geq 0$, we verify by (42) that

$$
\begin{aligned}
& \left\|f_{m}(x)-f_{n}(x)\right\| \\
& \quad \leq \sum_{k=n}^{m-1}\left\|f_{k+1}(x)-f_{k}(x)\right\| \\
& =\sum_{k=n}^{m-1} \frac{1}{3}\left(4^{2 k+1}-4^{k}\right) \\
& \quad \times\left\|f\left(\frac{x}{2^{k}}\right)-20 f\left(\frac{x}{2^{k+1}}\right)+64 f\left(\frac{x}{2^{k+2}}\right)\right\| \\
& \leq \sum_{k=n}^{m-1} \frac{64}{3}\left(4^{2 k+1}-4^{k}\right) \Phi\left(\frac{x}{2^{k+2}}\right)
\end{aligned}
$$

for all $x \in X$. Since the right-hand side of the above inequality tends to 0 as $n \rightarrow \infty$ by assumption, the sequence $\left\{f_{n}(x)\right\}$ is a convergent sequence. Now we define a mapping $H: X \rightarrow Y$ by

$$
\begin{aligned}
H(x)=\lim _{n \rightarrow \infty}[ & \frac{1}{3}\left(4^{2 n+1}-4^{n}\right) f\left(\frac{x}{2^{n}}\right) \\
& \left.-\frac{16}{3}\left(4^{2 n}-4^{n}\right) f\left(\frac{x}{2^{n+1}}\right)\right]
\end{aligned}
$$

for all $x \in X$. From (39) we figure out

$$
\begin{aligned}
\left\|D f_{n}(x, y)\right\| & \\
\leq & \frac{1}{3}\left(4^{2 n+1}-4^{n}\right)\left\|D f\left(\frac{x}{2^{n}}, \frac{y}{2^{n}}\right)\right\| \\
& +\frac{16}{3}\left(4^{2 n}-4^{n}\right)\left\|D f\left(\frac{x}{2^{n+1}}, \frac{y}{2^{n+1}}\right)\right\|
\end{aligned}
$$

$$
\begin{aligned}
\leq & \frac{1}{3}\left(4^{2 n+1}-4^{n}\right) \phi\left(\frac{x}{2^{n}}, \frac{y}{2^{n}}\right) \\
& +\frac{16}{3}\left(4^{2 n}-4^{n}\right) \phi\left(\frac{x}{2^{n+1}}, \frac{y}{2^{n+1}}\right)
\end{aligned}
$$

for all $n \in \mathbb{N}$ and for all $x, y \in X$. Letting $n \rightarrow \infty$ in the above inequality we see that $H$ satisfies (9). Letting $n \rightarrow \infty$ in (43) we finally obtain the result (40). This completes the proof.

From the previous Theorem 4, we obtain the following corollary concerning the stability of (9) immediately.

Corollary 6. Suppose that for some $\epsilon \geq 0$, a mapping $f: X \rightarrow$ $Y$ satisfies

$$
\|D f(x, y)\| \leq \epsilon
$$

for all $x, y \in X$. Then there exists a quartic and quadratic mapping $H: X \rightarrow Y$ such that

$$
\|f(x)-H(x)\| \leq \frac{17}{135} \epsilon
$$

for all $x \in X$.

\section{Stability of (9) under the Approximately Quartic Condition}

In the next part, we state and prove the Hyers-Ulam-Rassias stability of (9) under the approximately quartic condition.

Theorem 7. Let $\phi: X \times X \rightarrow \mathbb{R}^{+}$be a mapping satisfying

$$
\sum_{i=0}^{\infty} \frac{\phi\left(3^{i} x, 3^{i} y\right)}{81^{i}}<\infty
$$

for all $x, y \in X$ and let $\psi: X \rightarrow \mathbb{R}^{+}$be a mapping satisfying

$$
\sum_{i=0}^{\infty} \frac{\psi\left(3^{i} x\right)}{81^{i}}<\infty
$$

for all $x \in X$. If a mapping $f: X \rightarrow Y$ with $f(0)=0$ satisfies

$$
\|D f(x, y)\| \leq \phi(x, y)
$$

for all $x, y \in X$ and

$$
\|f(2 x)-16 f(-x)\| \leq \psi(x)
$$

for all $x \in X$, then there exists a unique quartic mapping $T$ : $X \rightarrow Y$ such that

$$
\begin{aligned}
\|f(x)-T(x)\| \\
\leq \sum_{i=1}^{\infty}\left\{\frac{1}{2}\left[\left(\frac{1}{81}\right)^{i}+\left(-\frac{1}{111}\right)^{i}\right] \Psi\left(3^{i-1} x\right)\right. \\
\left.+\frac{1}{2}\left[\left(\frac{1}{81}\right)^{i}-\left(-\frac{1}{111}\right)^{i}\right] \Psi\left(-3^{i-1} x\right)\right\}
\end{aligned}
$$


for all $x \in X$, where $\Psi(x)=\phi(x, x)+6 \psi(x)$. The mapping $T$ is given by

$$
T(x)=\lim _{n \rightarrow \infty} \frac{f\left(3^{n} x\right)}{81^{n}}
$$

for all $x \in X$.

Proof. It follows from (28) and (53) that we have

$$
\|f(3 x)+15 f(x)-96 f(-x)\| \leq \Psi(x)
$$

for all $x \in X$. Substituting $-x$ into $x$ in (56) yields

$$
\|f(-3 x)+15 f(-x)-96 f(x)\| \leq \Psi(-x)
$$

for all $x \in X$. Combining (56) and (57) to eliminate the term $f(-x)$ we obtain

$$
\begin{aligned}
\left\|f(x)-\frac{5}{2997} f(3 x)-\frac{32}{2997} f(-3 x)\right\| \\
\leq \frac{5}{2997}\|f(3 x)+15 f(x)-96 f(-x)\| \\
\quad+\frac{32}{2997}\|f(-3 x)+15 f(-x)-96 f(x)\| \\
\leq \frac{5}{2997} \Psi(x)+\frac{32}{2997} \Psi(-x)
\end{aligned}
$$

for all $x \in X$. Making use of induction arguments in (58) we have

$$
\begin{aligned}
\| f(x)-\frac{1}{2}\left[\left(\frac{1}{81}\right)^{n}+\left(-\frac{1}{111}\right)^{n}\right] f\left(3^{n} x\right) \\
-\frac{1}{2}\left[\left(\frac{1}{81}\right)^{n}-\left(-\frac{1}{111}\right)^{n}\right] f\left(-3^{n} x\right) \| \\
\leq \sum_{i=1}^{n}\left\{\frac{1}{2}\left[\left(\frac{1}{81}\right)^{i}+\left(-\frac{1}{111}\right)^{i}\right] \Psi\left(3^{i-1} x\right)\right. \\
\left.+\frac{1}{2}\left[\left(\frac{1}{81}\right)^{i}-\left(-\frac{1}{111}\right)^{i}\right] \Psi\left(-3^{i-1} x\right)\right\}
\end{aligned}
$$

for all $n \in \mathbb{N}$ and for all $x \in X$. Actually, (58) proves the validity of the inequality (59) for $n=1$. Using (56), (57), and the following relation

$$
\begin{array}{r}
f(x)-\frac{1}{2}\left[\left(\frac{1}{81}\right)^{n+1}+\left(-\frac{1}{111}\right)^{n+1}\right] f\left(3^{n+1} x\right) \\
-\frac{1}{2}\left[\left(\frac{1}{81}\right)^{n+1}-\left(-\frac{1}{111}\right)^{n+1}\right] f\left(-3^{n+1} x\right)
\end{array}
$$

$$
\begin{aligned}
= & f(x)-\frac{1}{2}\left[\left(\frac{1}{81}\right)^{n}+\left(-\frac{1}{111}\right)^{n}\right] f\left(3^{n} x\right) \\
& -\frac{1}{2}\left[\left(\frac{1}{81}\right)^{n}-\left(-\frac{1}{111}\right)^{n}\right] f\left(-3^{n} x\right) \\
& +\frac{1}{2}\left[\left(\frac{1}{81}\right)^{n+1}+\left(-\frac{1}{111}\right)^{n+1}\right] \\
& \times\left[-f\left(3^{n+1} x\right)-15 f\left(3^{n} x\right)+96 f\left(-3^{n} x\right)\right] \\
& +\frac{1}{2}\left[\left(\frac{1}{81}\right)^{n+1}-\left(-\frac{1}{111}\right)^{n+1}\right] \\
& \times\left[-f\left(-3^{n+1} x\right)-15 f\left(-3^{n} x\right)+96 f\left(3^{n} x\right)\right],
\end{aligned}
$$

one can easily verify (59) for $n+1$. It follows from (27) and (59) that

$$
\begin{aligned}
& \left\|f(x)-\frac{f\left(3^{n} x\right)}{81^{n}}\right\| \\
& \leq \sum_{i=1}^{n}\left\{\frac{1}{2}\left[\left(\frac{1}{81}\right)^{i}+\left(-\frac{1}{111}\right)^{i}\right] \Psi\left(3^{i-1} x\right)\right. \\
& \left.+\frac{1}{2}\left[\left(\frac{1}{81}\right)^{i}-\left(-\frac{1}{111}\right)^{i}\right] \Psi\left(-3^{i-1} x\right)\right\} \\
& +\frac{1}{6}\left[\left(\frac{1}{81}\right)^{n}-\left(-\frac{1}{111}\right)^{n}\right] \phi\left(0,3^{n} x\right)
\end{aligned}
$$

for all $n \in \mathbb{N}$ and for all $x \in X$. We show that the sequence $\left\{f\left(3^{n} x\right) / 81^{n}\right\}$ is a convergent sequence. For any integers $m, n$ with $m>n \geq 0$, we figure out

$$
\begin{aligned}
& \left\|\frac{f\left(3^{m}\right)}{81^{m}}-\frac{f\left(3^{n} x\right)}{81^{n}}\right\| \\
& =\frac{1}{81^{n}}\left\|\frac{f\left(3^{m-n} 3^{n} x\right)}{81^{m-n}}-f\left(3^{n} x\right)\right\| \\
& \leq \frac{1}{81^{n}} \sum_{i=1}^{m-n}\left\{\frac{1}{2}\left[\left(\frac{1}{81}\right)^{i}+\left(-\frac{1}{111}\right)^{i}\right] \Psi\left(3^{n+i-1} x\right)\right. \\
& \left.\quad+\frac{1}{2}\left[\left(\frac{1}{81}\right)^{i}-\left(-\frac{1}{111}\right)^{i}\right] \Psi\left(-3^{n+i-1} x\right)\right\} \\
& +\frac{1}{6 \cdot 81^{n}}\left[\left(\frac{1}{81}\right)^{m-n}-\left(-\frac{1}{111}\right)^{m-n}\right] \phi\left(0,3^{m-n} 3^{n} x\right)
\end{aligned}
$$

for all $x \in X$. Since the right-hand side of the inequality (62) tends to 0 as $n$ tends to infinity, the sequence $\left\{f\left(3^{n} x\right) / 81^{n}\right\}$ is a Cauchy sequence in $Y$. Now we define

$$
T(x)=\lim _{n \rightarrow \infty} \frac{f\left(3^{n} x\right)}{81^{n}}
$$

for all $x \in X$. Letting $n \rightarrow \infty$ in (61) we arrive at (54). 
Let us prove that the mapping $T: X \rightarrow Y$ satisfies (9). Replacing $x$ and $y$ by $3^{n} x$ and $3^{n} y$, respectively in (52) and dividing by $81^{n}$ yields

$$
81^{-n}\left\|D f\left(3^{n} x, 3^{n} y\right)\right\| \leq 81^{-n} \phi\left(3^{n} x, 3^{n} y\right)
$$

for all $n \in \mathbb{N}, x, y \in X$. Taking the limit as $n \rightarrow \infty$ in the above inequality, we see that $T$ satisfies (9) for all $x, y \in X$.

Finally we prove the uniqueness of the mapping $T$. Assume that there exists another quartic mapping $S: X \rightarrow Y$ which satisfies (9) and the inequality (54). Obviously, we have $T\left(3^{n} x\right)=81^{n} T(x)$ and $S\left(3^{n} x\right)=81^{n} S(x)$ for all $n \in \mathbb{N}, x \in X$. Hence it follows from (54) that

$$
\begin{aligned}
&\|T(x)-S(x)\| \\
& \leq 81^{-n}\left\{\left\|T\left(3^{n} x\right)-f\left(3^{n} x\right)\right\|+\left\|f\left(3^{n} x\right)-S\left(3^{n} x\right)\right\|\right\} \\
& \leq \frac{2}{81^{n}} \sum_{i=1}^{\infty}\left\{\frac{1}{2}\left[\left(\frac{1}{81}\right)^{i}+\left(-\frac{1}{111}\right)^{i}\right] \Psi\left(3^{i-1} x\right)\right. \\
&\left.+\frac{1}{2}\left[\left(\frac{1}{81}\right)^{i}-\left(-\frac{1}{111}\right)^{i}\right] \Psi\left(-3^{i-1} x\right)\right\}
\end{aligned}
$$

for all $x \in X$. Letting $n \rightarrow \infty$ in the above inequality, we immediately obtain the uniqueness of $T$.

Theorem 8. Let $\phi: X \times X \rightarrow \mathbb{R}^{+}$be a mapping satisfying

$$
\sum_{i=0}^{\infty} 111^{i} \phi\left(\frac{x}{3^{i}}, \frac{y}{3^{i}}\right)<\infty
$$

for all $x, y \in X$ and let $\psi: X \rightarrow \mathbb{R}^{+}$be a mapping satisfying

$$
\sum_{i=0}^{\infty} 111^{i} \psi\left(\frac{x}{3^{i}}\right)<\infty
$$

for all $x \in X$. If a mapping $f: X \rightarrow Y$ satisfies

$$
\|D f(x, y)\| \leq \phi(x, y)
$$

for all $x, y \in X$ and

$$
\|f(2 x)-16 f(-x)\| \leq \psi(x)
$$

for all $x \in X$, then there exists a unique quartic mapping $T$ : $X \rightarrow Y$ such that

$$
\begin{aligned}
\|f(x)-T(x)\| \\
\leq \sum_{i=0}^{\infty}\left\{\frac{1}{2}\left|-81^{i}+(-1)^{i-1} \cdot 111^{i}\right| \Psi\left(\frac{x}{3^{i+1}}\right)\right. \\
\left.\quad+\frac{1}{2}\left|-81^{i}-(-1)^{i-1} \cdot 111^{i}\right| \Psi\left(-\frac{x}{3^{i+1}}\right)\right\}
\end{aligned}
$$

for all $x \in X$. The mapping $T$ is given by

$$
T(x)=\lim _{n \rightarrow \infty} 81^{n} f\left(\frac{x}{3^{n}}\right)
$$

for all $x \in X$.
Proof. Replacing $x$ by $x / 3$ in (54) gives

$$
\left\|f(x)+15 f\left(\frac{x}{3}\right)-96 f\left(-\frac{x}{3}\right)\right\| \leq \Psi\left(\frac{x}{3}\right)
$$

for all $x \in X$. Using induction arguments in (72) we have

$$
\begin{aligned}
\| f(x)+\frac{1}{2}\left[-81^{n}+(-1)^{n-1} \cdot 111^{n}\right] f\left(\frac{x}{3^{n}}\right) \\
+\frac{1}{2}\left[-81^{n}-(-1)^{n-1} \cdot 111^{n}\right] f\left(-\frac{x}{3^{n}}\right) \| \\
\leq \sum_{i=0}^{n-1}\left\{\frac{1}{2}\left|-81^{i}+(-1)^{i-1} \cdot 111^{i}\right| \Psi\left(\frac{x}{3^{i+1}}\right)\right. \\
\left.\quad+\frac{1}{2}\left|-81^{i}-(-1)^{i-1} \cdot 111^{i}\right| \Psi\left(-\frac{x}{3^{i+1}}\right)\right\}
\end{aligned}
$$

for all $n \in \mathbb{N}$ and for all $x \in X$. It follows from (27) and (73) that

$$
\begin{gathered}
\left\|f(x)-81^{n} f\left(\frac{x}{3^{n}}\right)\right\| \\
\leq \sum_{i=0}^{n-1}\left\{\frac{1}{2}\left|-81^{i}+(-1)^{i-1} \cdot 111^{i}\right| \Psi\left(\frac{x}{3^{i+1}}\right)\right. \\
\left.+\frac{1}{2}\left|-81^{i}-(-1)^{i-1} \cdot 111^{i}\right| \Psi\left(-\frac{x}{3^{i+1}}\right)\right\} \\
+\frac{1}{6}\left|-81^{i}-(-1)^{i-1} \cdot 111^{i}\right| \phi\left(0, \frac{x}{3^{n}}\right)
\end{gathered}
$$

for all $n \in \mathbb{N}$ and for all $x \in X$. The rest of the proof is similar to the proof of Theorem 7 .

By Theorem 7, we have the following corollary immediately.

Corollary 9. Suppose that for some $\epsilon \geq 0, \delta \geq 0$, a mapping $f: X \rightarrow Y$ satisfies

$$
\|D f(x, y)\| \leq \epsilon
$$

for all $x, y \in X$ and

$$
\|f(2 x)-16 f(-x)\| \leq \delta
$$

for all $x \in X$. Then there exists a unique quartic mapping $T$ : $X \rightarrow Y$ such that

$$
\|f(x)-T(x)\| \leq \frac{\epsilon+6 \delta}{80}
$$

for all $x \in X$.

\section{Stability of (9) under the Approximately Quadratic Condition}

Now we state and prove the Hyers-Ulam-Rassias stability of (9) under the approximately quadratic condition. 
Theorem 10. Let $\phi: X \times X \rightarrow \mathbb{R}^{+}$be a mapping satisfying

$$
\sum_{i=0}^{\infty} \frac{\phi\left(3^{i} x, 3^{i} y\right)}{9^{i}}<\infty
$$

for all $x, y \in X$ and let $\psi: X \rightarrow \mathbb{R}^{+}$be a mapping satisfying

$$
\sum_{i=0}^{\infty} \frac{\psi\left(3^{i} x\right)}{9^{i}}<\infty
$$

for all $x \in X$. If a mapping $f: X \rightarrow Y$ with $f(0)=0$ satisfies

$$
\|D f(x, y)\| \leq \phi(x, y)
$$

for all $x, y \in X$ and

$$
\|f(2 x)-4 f(-x)\| \leq \psi(x)
$$

for all $x \in X$, then there exists a unique quadratic mapping $\mathrm{Q}: X \rightarrow Y$ such that

$$
\begin{aligned}
\|f(x)-Q(x)\| \\
\leq \sum_{i=1}^{\infty}\left\{\frac{1}{2}\left[\left(\frac{1}{9}\right)^{i}+\left(-\frac{1}{39}\right)^{i}\right] \Psi\left(3^{i-1} x\right)\right. \\
\left.+\frac{1}{2}\left[\left(\frac{1}{9}\right)^{i}-\left(-\frac{1}{39}\right)^{i}\right] \Psi\left(-3^{i-1} x\right)\right\}
\end{aligned}
$$

for all $x \in X$. The mapping $Q$ is defined by

$$
Q(x)=\lim _{n \rightarrow \infty} \frac{f\left(3^{n} x\right)}{9^{n}}
$$

for all $x \in X$.

Proof. It follows from (28) and (81) that we have

$$
\|f(3 x)+15 f(x)-24 f(-x)\| \leq \Psi(x)
$$

for all $x \in X$. Substituting $-x$ for $x$ in (84) yields

$$
\|f(-3 x)+15 f(-x)-24 f(x)\| \leq \Psi(-x)
$$

for all $x \in X$. Combining (84) and (85) to eliminate the term $f(-x)$ we obtain

$$
\begin{aligned}
\left\|f(x)-\frac{5}{117} f(3 x)-\frac{8}{117} f(-3 x)\right\| \\
\leq \frac{5}{117}\|-f(3 x)-15 f(x)+24 f(-x)\| \\
\quad+\frac{8}{117}\|-f(-3 x)-15 f(-x)+24 f(x)\| \\
\leq \frac{5}{117} \Psi(x)+\frac{8}{117} \Psi(-x)
\end{aligned}
$$

for all $x \in X$. Making use of induction arguments in (86) we have

$$
\begin{aligned}
\| f(x)-\frac{1}{2}\left[\left(\frac{1}{9}\right)^{n}+\left(-\frac{1}{39}\right)^{n}\right] f\left(3^{n} x\right) \\
-\frac{1}{2}\left[\left(\frac{1}{9}\right)^{n}-\left(-\frac{1}{39}\right)^{n}\right] f\left(-3^{n} x\right) \| \\
\leq \sum_{i=1}^{n}\left\{\frac{1}{2}\left[\left(\frac{1}{9}\right)^{i}+\left(-\frac{1}{39}\right)^{i}\right] \Psi\left(3^{i-1} x\right)\right. \\
\left.+\frac{1}{2}\left[\left(\frac{1}{9}\right)^{i}-\left(-\frac{1}{39}\right)^{i}\right] \Psi\left(-3^{i-1} x\right)\right\}
\end{aligned}
$$

for all $n \in \mathbb{N}$ and for all $x \in X$. It follows from (86) and (87) that

$$
\begin{aligned}
& \left\|f(x)-\frac{f\left(3^{n} x\right)}{9^{n}}\right\| \\
& \leq \sum_{i=1}^{n}\left\{\frac{1}{2}\left[\left(\frac{1}{9}\right)^{i}+\left(-\frac{1}{39}\right)^{i}\right] \Psi\left(3^{i-1} x\right)\right. \\
& \left.+\frac{1}{2}\left[\left(\frac{1}{9}\right)^{i}-\left(-\frac{1}{39}\right)^{i}\right] \Psi\left(-3^{i-1} x\right)\right\} \\
& +\frac{1}{6}\left[\left(\frac{1}{9}\right)^{n}-\left(-\frac{1}{39}\right)^{n}\right] \phi\left(0,3^{n} x\right)
\end{aligned}
$$

for all $n \in \mathbb{N}, x \in X$. From (88) one can easily show that the sequence $\left\{f\left(3^{n} x\right) / 9^{n}\right\}$ is a Cauchy sequence in $Y$. Define a mapping

$$
Q(x)=\lim _{n \rightarrow \infty} \frac{f\left(3^{n} x\right)}{9^{n}}
$$

for all $x \in X$. It follows from (80) and (88) that we verify the mapping $Q$ is the unique mapping satisfying (9) and (82). Letting $n \rightarrow \infty$ in (88) we have the result (82).

Theorem 11. Let $\phi: X \times X \rightarrow \mathbb{R}^{+}$be a mapping satisfying

$$
\sum_{i=0}^{\infty} 39^{i} \phi\left(\frac{x}{3^{i}}, \frac{y}{3^{i}}\right)<\infty
$$

for all $x, y \in X$ and let $\psi: X \rightarrow \mathbb{R}^{+}$be a mapping satisfying

$$
\sum_{i=0}^{\infty} 39^{i} \psi\left(\frac{x}{3^{i}}\right)<\infty
$$

for all $x \in X$. If a mapping $f: X \rightarrow Y$ satisfies

$$
\|D f(x, y)\| \leq \phi(x, y)
$$

for all $x, y \in X$ and

$$
\|f(2 x)-4 f(-x)\| \leq \psi(x)
$$


for all $x \in X$, then there exists a unique quadratic mapping $Q: X \rightarrow Y$ such that

$$
\begin{aligned}
&\|f(x)-Q(x)\| \\
& \leq \sum_{i=1}^{\infty}\left\{\frac{1}{2}\left|-9^{i}+(-1)^{i-1} \cdot 39^{i}\right| \Psi\left(\frac{x}{3^{i+1}}\right)\right. \\
&\left.+\frac{1}{2}\left|-9^{i}-(-1)^{i-1} \cdot 39^{i}\right| \Psi\left(-\frac{x}{3^{i+1}}\right)\right\}
\end{aligned}
$$

for all $x \in X$. The mapping $Q$ is given by

$$
Q(x)=\lim _{n \rightarrow \infty} 9^{n} f\left(\frac{x}{3^{n}}\right)
$$

for all $x \in X$.

Proof. Putting $x=x / 3$ in (84) gives

$$
\left\|f(x)+15 f\left(\frac{x}{3}\right)-24 f\left(-\frac{x}{3}\right)\right\| \leq \Psi\left(\frac{x}{3}\right)
$$

for all $x \in X$. Making use of induction arguments in (96) we have

$$
\begin{aligned}
\| f(x)+\frac{1}{2}\left[-9^{n}+(-1)^{n-1} \cdot 39^{n}\right] f\left(\frac{x}{3^{n}}\right) \\
+\frac{1}{2}\left[-9^{n}-(-1)^{n-1} \cdot 39^{n}\right] f\left(-\frac{x}{3^{n}}\right) \| \\
\leq \sum_{i=0}^{n-1}\left\{\frac{1}{2}\left|-9^{i}+(-1)^{i-1} \cdot 39^{i}\right| \Psi\left(\frac{x}{3^{i+1}}\right)\right. \\
\left.+\frac{1}{2}\left|-9^{i}-(-1)^{i-1} \cdot 39^{i}\right| \Psi\left(-\frac{x}{3^{i+1}}\right)\right\}
\end{aligned}
$$

for all $n \in \mathbb{N}$ and for all $x \in X$. It follows from (27) and (73) that

$$
\begin{gathered}
\left\|f(x)-9^{n} f\left(\frac{x}{3^{n}}\right)\right\| \\
\leq \sum_{i=0}^{n-1}\left\{\frac{1}{2}\left|-9^{i}+(-1)^{i-1} \cdot 39^{i}\right| \Psi\left(\frac{x}{3^{i+1}}\right)\right. \\
\left.+\frac{1}{2}\left|-9^{i}-(-1)^{i-1} \cdot 39^{i}\right| \Psi\left(-\frac{x}{3^{i+1}}\right)\right\} \\
+\frac{1}{6}\left|-9^{i}-(-1)^{i-1} \cdot 39^{i}\right| \phi\left(0, \frac{x}{3^{n}}\right)
\end{gathered}
$$

for all $n \in \mathbb{N}$ and for all $x \in X$. The rest of the proof goes through in the similar way as that of the proof of Theorem 10.

It follows from Theorem 10 that we obtain the following corollary immediately.

Corollary 12. Suppose that for some $\epsilon \geq 0, \delta \geq 0$, a mapping $f: X \rightarrow Y$ satisfies

$$
\|D f(x, y)\| \leq \epsilon
$$

for all $x, y \in X$ and

$$
\|f(2 x)-4 f(-x)\| \leq \delta
$$

for all $x \in X$, for all $x, y \in X$, and for some $\delta \geq 0$. Then there exists a unique quartic mapping $T: X \rightarrow Y$ such that

$$
\|f(x)-Q(x)\| \leq \frac{\epsilon+6 \delta}{8}
$$

for all $x \in X$.

\section{Conflict of Interests}

The authors declare that there is no conflict of interests regarding the publication of this paper.

\section{References}

[1] S. M. Ulam, Problems in Modern Mathematics, John Wiley \& Sons, New York, NY, USA, 1964.

[2] D. H. Hyers, "On the stability of the linear functional equation," Proceedings of the National Academy of Sciences of the United States of America, vol. 27, pp. 222-224, 1941.

[3] T. Aoki, "On the stability of the linear transformation in Banach spaces," Journal of the Mathematical Society of Japan, vol. 2, pp. 64-66, 1950.

[4] T. M. Rassias, "On the stability of the linear mapping in Banach spaces," Proceedings of the American Mathematical Society, vol. 72, no. 2, pp. 297-300, 1978.

[5] G. Z. Eskandani, P. Găvruţa, J. M. Rassias, and R. Zarghami, "Generalized Hyers-Ulam stability for a general mixed functional equation in quasi- $\beta$-normed spaces," Mediterranean Journal of Mathematics, vol. 8, no. 3, pp. 331-348, 2011.

[6] G. Z. Eskandani, J. M. Rassias, and P. Găvruța, "Generalized Hyers-Ulam stability for a general cubic functional equation in quasi- $\beta$-normed spaces," Asian-European Journal of Mathematics, vol. 4, no. 3, pp. 413-425, 2011.

[7] J. M. Rassias, "On approximation of approximately linear mappings by linear mappings," Journal of Functional Analysis, vol. 46, no. 1, pp. 126-130, 1982.

[8] J. M. Rassias and H.-M. Kim, "Approximate $(m, n)$-CauchyJensen mappings in quasi- $\beta$-normed spaces," Journal of Computational Analysis and Applications, vol. 16, pp. 346-358, 2014.

[9] K. Ravi, M. Arunkumar, and J. M. Rassias, "Ulam stability for the orthogonally general Euler-Lagrange type functional equation," International Journal of Mathematics and Statistics, vol. 3, no. 8, pp. 36-46, 2008.

[10] P. Găvruţa, "A generalization of the Hyers-Ulam-Rassias stability of approximately additive mappings," Journal of Mathematical Analysis and Applications, vol. 184, no. 3, pp. 431-436, 1994.

[11] L. Cădariu and V. Radu, "Fixed points and the stability of Jensen's functional equation," Journal of Inequalities in Pure and Applied Mathematics, vol. 4, no. 1, article 4, 2003.

[12] L. Cădariu, L. Găvruţa, and P. Găvruţa, "Fixed points and generalized HYers-Ulam stability," Abstract and Applied Analysis, vol. 2012, Article ID 712743, 10 pages, 2012.

[13] S. Czerwik, Functional Equations and Inequalities in Several Variables, World Scientific Publishing, River Edge, NJ, USA, 2002. 
[14] D. H. Hyers, G. Isac, and T. M. Rassias, Stability of Functional Equations in Several Variables, Birkhäuser, Boston, Mass, USA, 1998.

[15] S.-M. Jung, Hyers-Ulam-Rassias Stability of Functional Equations in Mathematical Analysis, Hadronic Press, Palm Harbor, Fla, USA, 2001.

[16] S.-M. Jung, Hyers-Ulam-Rassias Stability of Functional Equations in Nonlinear Analysis, Springer Optimization and Its Applications, Springer, New York, NY, USA, 2011.

[17] P. Kannappan, Functional Equations and Inequalities with Applications, Springer, New York, NY, USA, 2009.

[18] P. K. Sahoo and P. Kannappan, Introduction to Functional Equations, CRC Press, Boca Raton, Fla, USA, 2011.

[19] X. Zhao, X. Yang, and C. Pang, "Solution and stability of a general mixed type cubic and quartic functional equation," Journal of Function Spaces and Applications, vol. 2013, Article ID 673810, 8 pages, 2013.

[20] C. Borelli and G. L. Forti, "On a general Hyers-Ulam stability result," International Journal of Mathematics and Mathematical Sciences, vol. 18, no. 2, pp. 229-236, 1995.

[21] S. Czerwik, "On the stability of the quadratic mapping in normed spaces," Abhandlungen aus dem Mathematischen Seminar der Universität Hamburg, vol. 62, pp. 59-64, 1992.

[22] F. Skof, "Local properties and approximation of operators," Rendiconti del Seminario Matematico e Fisico di Milano, vol. 53, pp. 113-129, 1983.

[23] J. M. Rassias, "Solution of the Ulam stability problem for quartic mappings," Glasnik Matematicki. Serija III, vol. 34, no. 2, pp. 243-252, 1999.

[24] J. K. Chung and P. K. Sahoo, "On the general solution of a quartic functional equation," Bulletin of the Korean Mathematical Society, vol. 40, no. 4, pp. 565-576, 2003.

[25] S. H. Lee, S. M. Im, and I. S. Hwang, "Quartic functional equations," Journal of Mathematical Analysis and Applications, vol. 307, no. 2, pp. 387-394, 2005.

[26] A. Najati, "On the stability of a quartic functional equation," Journal of Mathematical Analysis and Applications, vol. 340, no. 1, pp. 569-574, 2008.

[27] Y.-S. Lee and S.-Y. Chung, "Stability of quartic functional equations in the spaces of generalized functions," Advances in Difference Equations, vol. 2009, Article ID 838347, 16 pages, 2009.

[28] H.-M. Kim, "On the stability problem for a mixed type of quartic and quadratic functional equation," Journal of Mathematical Analysis and Applications, vol. 324, no. 1, pp. 358-372, 2006.

[29] M. E. Gordji, S. Abbaszadeh, and C. Park, "On the stability of a generalized quadratic and quartic type functional equation in quasi-Banach spaces," Journal of Inequalities and Applications, vol. 2009, Article ID 153084, 26 pages, 2009.

[30] J. Aczél and J. Dhombres, Functional Equations in Several Variables, Cambridge University Press, Cambridge, UK, 1989. 


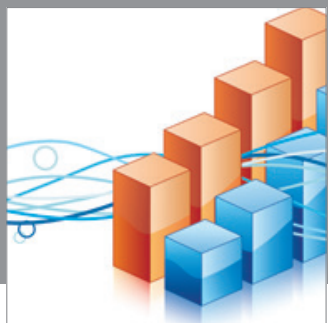

Advances in

Operations Research

mansans

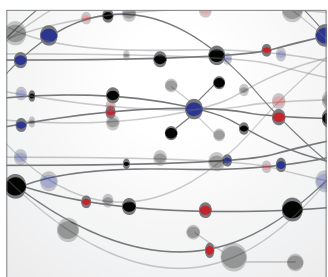

The Scientific World Journal
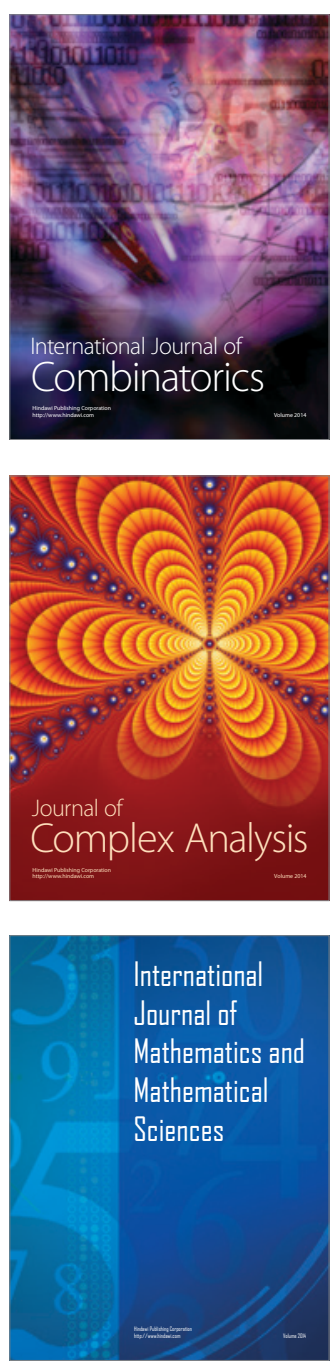
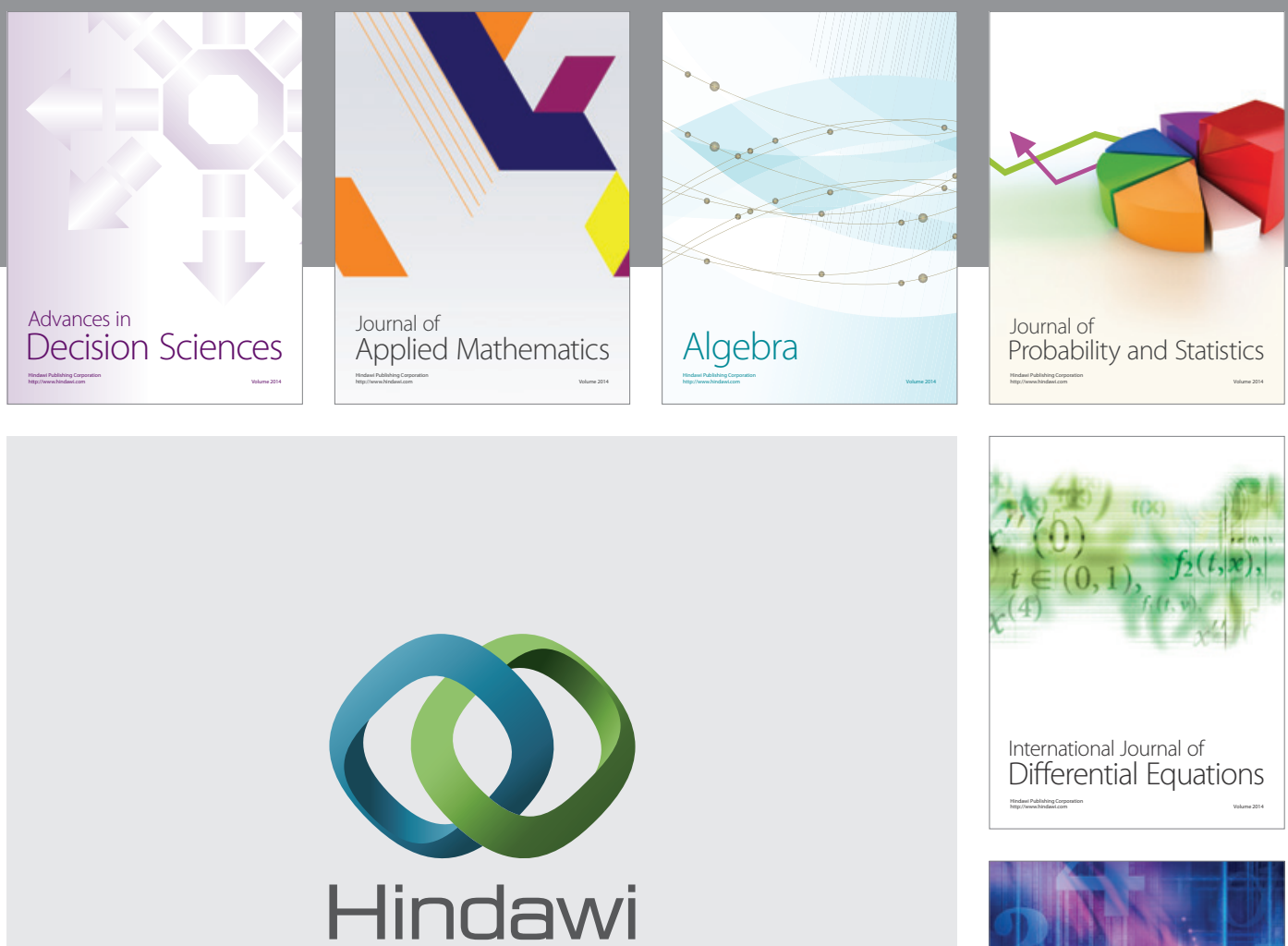

Submit your manuscripts at http://www.hindawi.com
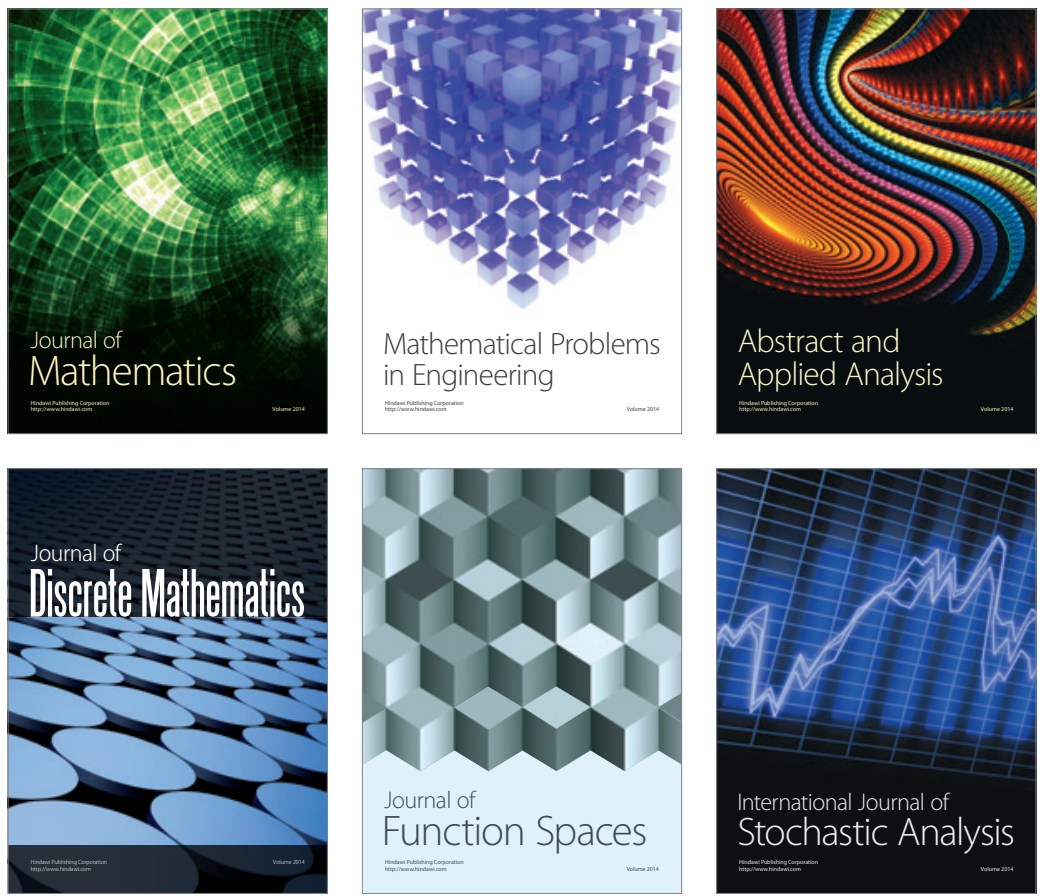

Journal of

Function Spaces

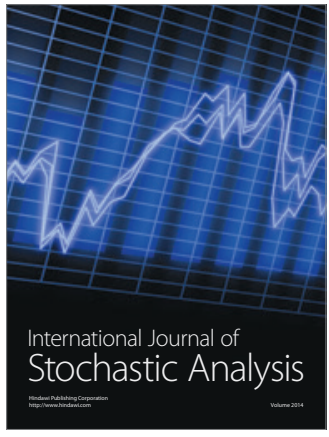

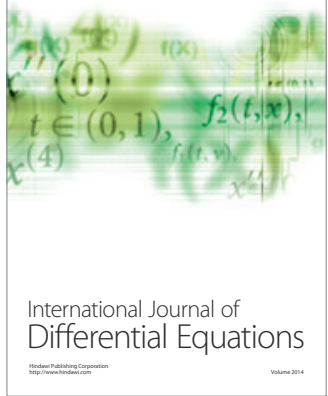
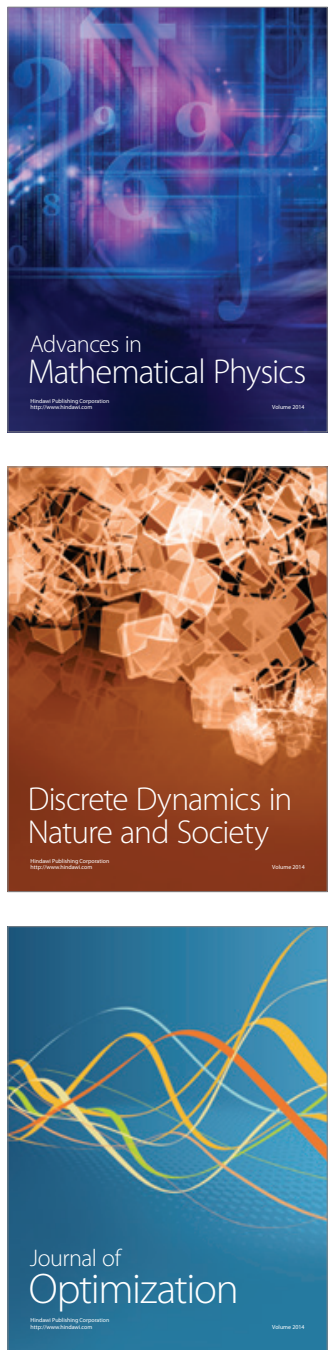